\title{
Permeation Behavior of Gas Molecules in Ultramicropore of Molecular Sieving Carbon Membranes
}

\author{
H. Okabe ${ }^{1,2}$, H. Takagi ${ }^{1}$, H. Hatori ${ }^{1, *}$, A. Suzuki $^{2}$ and T. Terai ${ }^{2}$ \\ ${ }^{1}$ National Institute of Advanced Industrial Science and Technology, 16-1 Onogawa, Tsukuba 305-8569, Japan. \\ ${ }^{2}$ University of Tokyo, 2-11-16 Yayoi, Bunkyo-ku, Tokyo 113-8656, Japan. \\ Fax: +81-29-861-8408, e-mail: h.hatori@aist.go.jp
}

Permeation behavior of gas molecules in ultramicropore was investigated by using molecular sieving carbon membranes. Hydrogen and hydrogen isotope (deuterium), which have the same molecular size but different mass, were used in order to discuss the diffusion mechanism. Although the temperature dependence of permeation coefficient apparently obeyed the Knudsen diffusion in the membranes which have wider ultramicropores, the deviation of the separation factor of hydrogen and deuterium from the theoretical value suggested coexistence of the other diffusion mechanism. With decreasing ultramicropore size, the permeation behavior of hydrogen deviated from Knudsen diffusion, indicating surface diffusion dominantly occurred. The stronger interaction of deuterium in ultramicropores was shown by the difference of activation energy of surface diffusion between hydrogen and deuterium. Apparent solubility factor, assuming surface diffusion, clearly showed that the deuterium is considerably adsorbed in ultramicropores more than hydrogen even at ambient temperatures.

Key words: carbon membranes, molecular sieves, Knudsen diffusion, surface diffusion, activation energy

\section{INTRODUCTION}

Inorganic membranes made of zeolite $[1,2]$, silica [3-5] or carbon [6-12], which have been actively studied in last decade, are considered for the separation process at high temperatures since they have high thermal- and corrosion-resistance and their properties of gas separation are quite different from those of polymer membranes. The characteristic properties of the inorganic membranes are attributable to the very narrow pore channels in sub-nanometer scale, in which interaction between the solid surface and gas molecules determines the separation ability. We have reported the control of size distribution of ultramicropores (micropores less than $0.5 \mathrm{~nm}$ in diameter) and the gas permeability in molecular sieving carbon membranes (MSCMs) that are easily prepared from polyimide by simple heat-treatment in inert atmosphere $[6,13]$. The membranes which have ultramicropores with the size of about $4-5 \mathrm{~nm}$ show a high selectivity on $\mathrm{CO}_{2}$ permeation.

Recently, development of polymer electrolyte fuel cells is very active as an alternative power source especially for vehicles. Hydrogen does not exist in the nature as energy resource and so far is produced from fossil fuel basically. In case of polymer electrolyte fuel cell, a serious poison of the catalyst takes place by coexistence of carbon monoxide in the hydrogen fuel gas; even a few tens parts per million $C O$ causes a substantial degradation on the cell performance $[14,15]$. We have reported the hydrogen purification ability of MSCMs with the ultramicropore size of about $3 \mathrm{~nm}$ [16]. The MSCMs had quite high $\mathrm{H}_{2} / \mathrm{CO}$ ratio of permeability over 5000 , suggesting that the membranes can reduce the $C O$ content in a reformed gas from $1 \%$ to a few ppm.

Despite the unique gas-separation ability of inorganic membranes, the permeation mechanism of gases in very narrow micropore has not been fully demonstrated. Although carbon membranes have a disadvantage in the oxidation-resistance if compared with zeolite ones which is actively investigated from the viewpoint of application to the gas reforming system, the MSCMs are advantageous in preparation of pinhole-less membranes because of their intrinsically amorphous microtexture without clear grain boundary [16]. Namely, the fundamental study on the diffusion mechanism inside of micropores is easy and appropriate in carbon membranes having homogeneity in the pore structure. In the present study, the adsorption and diffusion behavior of hydrogen, which has very weak interaction with carbon surface, was investigated by using MSCMs with ultramicropore, the size of which is very close to the diameter of hydrogen molecules.

\section{EXPERIMENTAL}

\subsection{Sample preparation}

A commercially available polyimide film (Kapton ${ }^{\Phi}$, Toray-DuPont Ltd.) with the thickness of $0.125 \mathrm{~mm}$ was used as a precursor of MSCMs. The film was heat-treated in flowing high purity nitrogen (99.99995\%), giving MSCMs with the thickness of $0.1 \mathrm{~mm}$ after carbonization. The pore size can be controlled by heat-treatment temperatures (HTTs) because the pore size tends to shrink at higher HTTs. $[13,16]$. The MSCM specimens are represented by the final heat-treatment temperatures, e.g. HTT1173K. The morphology and pore size 
distribution of MSCMs used in this study have already been reported in [16]. The pore size distribution of the MSCMs was estimated by adsorption at $298 \mathrm{~K}$ using gas molecules with different minimum dimension such as $\mathrm{CO}_{2}$ $(0.33 \mathrm{~nm})$, ethane $(0.40 \mathrm{~nm})$ and so on. The MSCM1173K has ultramicropores with the size between 0.33 and $0.40 \mathrm{~nm}$, while MSCM1373K can not admit $\mathrm{CO}_{2}$, indicating the ultramicropore size less than $0.33 \mathrm{~nm}$.

\subsection{Permeability measurement}

Gas permeability through a MSCM was measured after the treatment in $10^{-4} \mathrm{~Pa}$ at $383 \mathrm{~K}$. The feed gas pressure, $\mathrm{P}_{\mathrm{l}}$, of about $200 \mathrm{kPa}$ was loaded from one side of the membrane and the increase of pressure in another side was measured until the pressure reached $0.5 \mathrm{kPa}$. Permeability constant, $P$ is defined by eq. (1):

$$
\boldsymbol{Q}=\boldsymbol{P} \frac{\boldsymbol{p}_{i}}{\boldsymbol{L}}
$$

where $\mathrm{Q}\left(\mathrm{mol} \cdot \mathrm{m}^{-2} \cdot \mathrm{s}^{-1}\right)$ is a molar rate of transport of pure gas per unit area and $L$ is the membrane thickness. Diffusion coefficient, $D\left(\mathrm{~m}^{2} \cdot \mathrm{s}^{-1}\right)$ was given from the time lag, $\theta$ [17] by eq. (2):

$$
D=\frac{L^{2}}{6 \theta}
$$

\subsection{Adsorption isotherm measurement}

Adsorption isotherms of hydrogen and deuterium were measured by commercial adsorption apparatus, Belsorp 28SA (BEL Japan Inc.) at 77K.

\section{RESULTS AND DISCUSSION}

Permeation measurements were performed for hydrogen and deuterium which has the same molecular size but different mass. When permeation takes place without any interaction force between gas molecules and carbon surface in pores, the diffusion coefficient, $D$, is proportional to the $\mathrm{T}^{1 / 2}$, square root of the measurement temperature (Knudsen diffusion). The permeability $P$ is proportional to the $T^{-1 / 2}$ in Knudsen diffusion as well. On the other hand, if surface diffusion occurs in ultramicropores, a flux is represented by the Fick's diffusion law, and the diffusion coefficient is expressed by the frequency factor and activation energy, $E$.

In Fig. 1 and $2, \mathrm{P}$ and $\mathrm{D}$ are plotted against $T^{-1 / 2}$ and $T^{1 / 2}$, respectively. As coefficient of determination, $\mathbf{R}^{2}$ shows, good correlation was given on permeation of both hydrogen and deuterium for the samples of HTT1173K (not shown in the figure) and $1273 \mathrm{~K}$ (Fig. 1a). The correlation between $\mathrm{D}$ and $\mathrm{T}^{1 / 2}$ was good as well. These results indicate that the Knudsen diffusion occurs predominantly in the membranes. However, the ratio of $P_{\mathrm{H}_{2}}$ and $\mathrm{P}_{\mathrm{D} 2}, \mathrm{P}_{\mathrm{H} 2} / \mathrm{P}_{\mathrm{D} 2}$, and $\mathrm{D}_{\mathrm{H} 2}$ and $\mathrm{D}_{\mathrm{D} 2}$, $\mathrm{D}_{\mathrm{H} 2} / \mathrm{D}_{\mathrm{D} 2}$, was less than 1.41 , the theoretical value
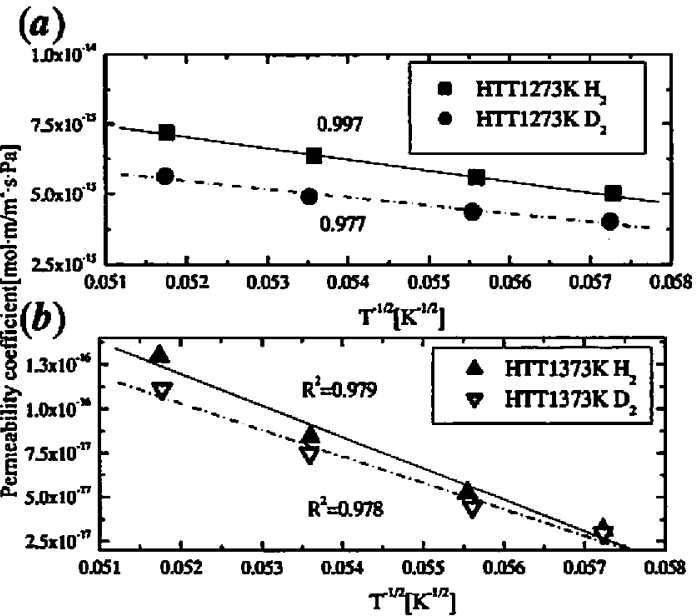

Fig.1. Temperature dependence of permeability coefficient of $\mathrm{H}_{2}$ and $\mathrm{D}_{2}$ in MSCMs; $\mathrm{P}-\mathrm{T}^{-1 / 2}$ in (a) HTT1273K and (b) HTT1373K.

(a)
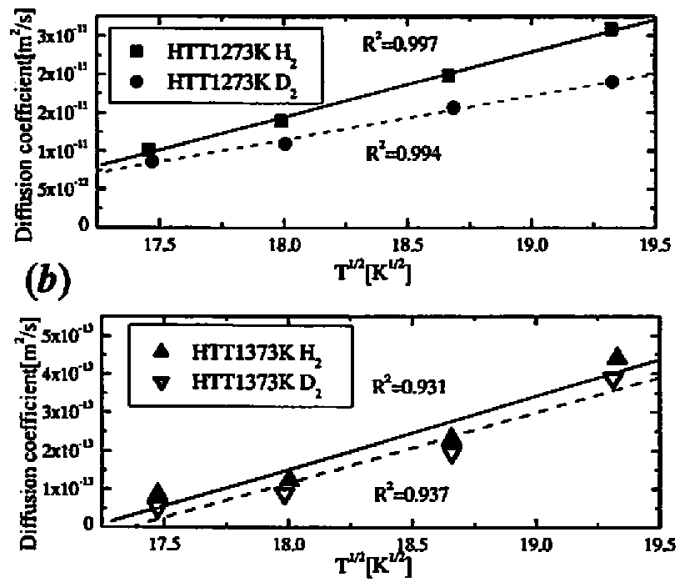

Fig. 2. Temperature dependence of diffusion coefficient of $\mathrm{H}_{2}$ and $\mathrm{D}_{2}$ in MSCMs; D-T ${ }^{1 / 2}$ in (a) HTT1273K and (b) HTT1373K.

assuming Knudsen diffusion mechanism (Fig. 3). It is thought that the diffusion in ultramicropores even in HTT1173K and HTT1273K occurs by not only Knudsen diffusion but also the other mechanism which is not based on the simple molecular motion.

On the other hand, the P-T $T^{-1 / 2}$ and D-T $T^{1 / 2}$ plots showed a bad correlation (Fig. $1 b$ and $2 b$ ) for HTT1373K which has narrower ultramicropores than HTT1173K and HTT1273K. The temperature dependence of $D_{H 2} / D_{D 2}$ is more characteristic, as shown in Fig. 3a and $b$, though the separation factor $\mathbf{P}_{\mathrm{H} 2} / \mathbf{P}_{\mathrm{D} 2}$, is almost constant. The behavior is explainable if surface diffusion is assumed to govern the mass transfer in ultramicropores in HTT1373K in which higher physisorption potentials are expected by the narrow pores with the size comparable to hydrogen. When the mass transfer takes place by surface diffusion mechanism, the relation of $P=D S$ can be applied, 
(a)
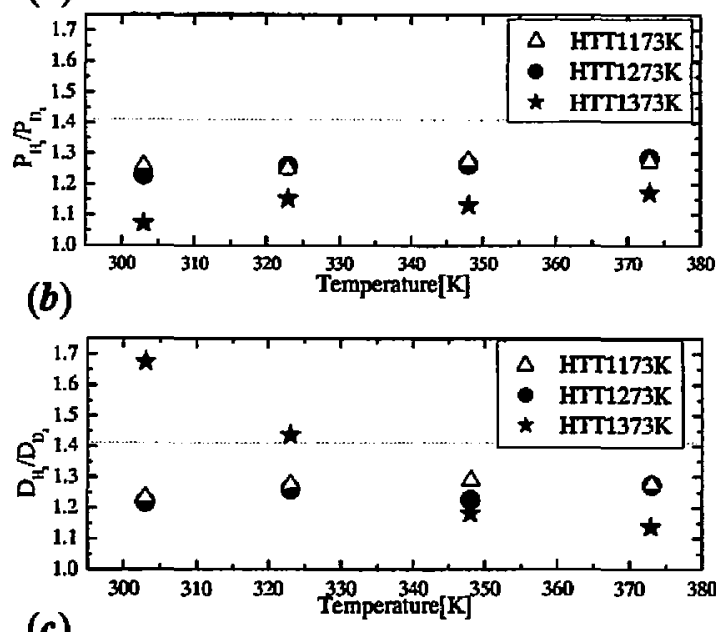

(c)

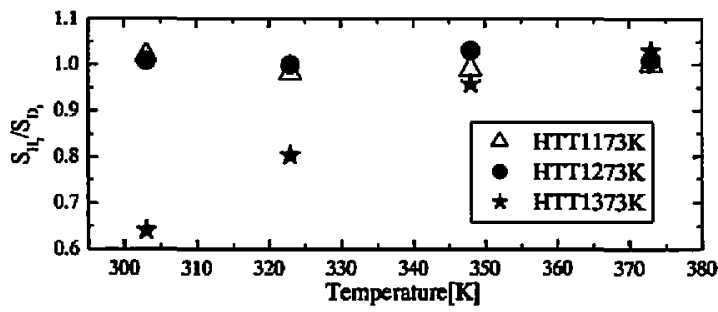

Fig. 3. Temperature dependence of the ratio of (a) $\mathrm{P}_{\mathrm{H} 2} / \mathrm{P}_{\mathrm{D} 2}$, (b) $\mathrm{D}_{\mathrm{H} 2} / \mathrm{D}_{\mathrm{D} 2}$ and (c) $\mathrm{S}_{\mathrm{H} 2} / \mathrm{S}_{\mathrm{D} 2}$ which is calculated from the ratio of $\mathrm{P}_{\mathrm{H} 2} / \mathrm{P}_{\mathrm{D} 2}$ and $\mathrm{D}_{\mathrm{H} 2} / \mathrm{D}_{\mathrm{D} 2}$.

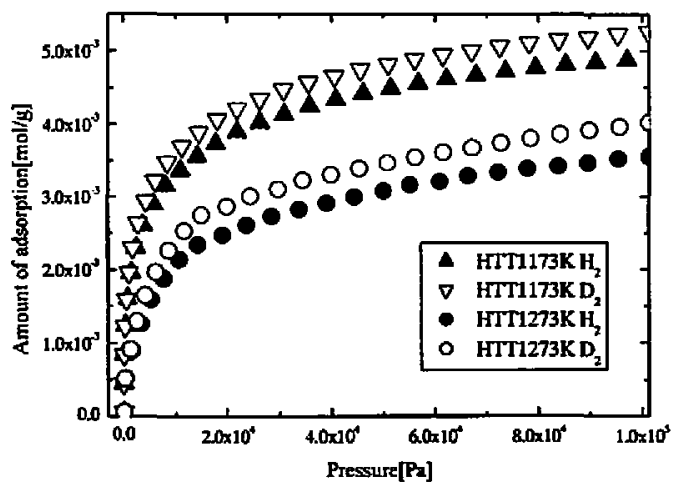

Fig.4. Adsorption isotherms of hydrogen and deuterium at $77 \mathrm{~K}$.

where $S$ is the apparent solubility coefficient of gas in the bulk membrane [16]. The ratio of the solubility factor, $\mathrm{S}_{\mathrm{H} 2} / \mathrm{S}_{\mathrm{D} 2}$, calculated from $\mathrm{P}_{\mathrm{H} 2} / \mathrm{P}_{\mathrm{D} 2}$ divided by $\mathrm{D}_{\mathrm{H} 2} / \mathrm{D}_{\mathrm{D} 2}$, showed temperature dependence; the lower the measurement temperature was, the higher the solubility coefficient of deuterium.

It is known that the interaction of hydrogen and deuterium with the solid surface is different and the molecules show some different behavior of physisorption. It is quite difficult to measure accurate physisorption isotherms of hydrogen at ambient temperatures because of the small adsorption.Therefore, the physisorption isotherms were measured at $77 \mathrm{~K}$ and demonstrated in Fig. 4. As is clearly shown in the isotherms, the amount of deuterium physisorbed is larger than that of hydrogen in the MSCMs. Considering from the difference of the physisorption ability, deuterium has stronger interaction with carbon surface in ultramicropores. The effect is considered to be more remarkable at low temperatures, and hence the ratio of apparent solubility factor for bulk membrane, $S_{\mathrm{H}_{2}} / \mathrm{S}_{\mathrm{D} 2}$, became lower at lower permeation temperatures (Fig. $3 \mathrm{c}$ ) because of larger amount of deuterium adsorbed than hydrogen. On the other hand, the diffusivity of deuterium became slow relatively, as shown by the ratio of diffusion coefficient in Fig. $3 b$, by the stronger effect of physisorption in the narrow ultramicropores.

Arrhenius plots of diffusion coefficient are shown in Fig. 5. Assuming the surface diffusion mechanism, activation energy was determined and shown in the figure. The activation energy was remarkably higher in HTT1373K if compared to the samples treated at lower HTTs which have wider ultramicropores. In addition, activation energy of deuterium is considerably higher than that of hydrogen in HTT1373K. These results support the discussions above; deuterium has stronger interaction with carbon surface in ultramicropores and the $D_{D_{2}}$ is smaller but $S_{D 2}$ is larger than those of hydrogen, respectively.

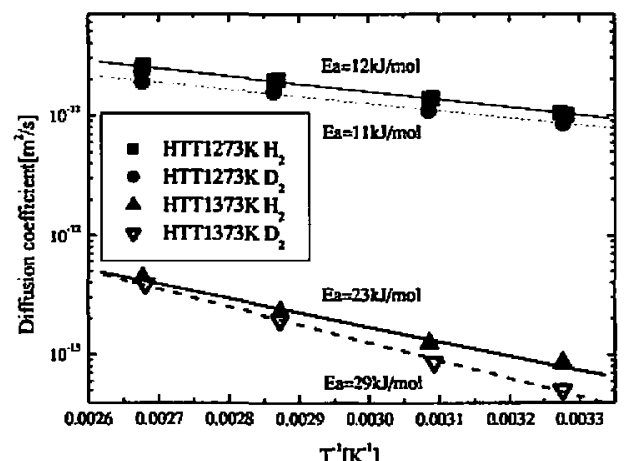

Fig. 5. Arrhenius plots of hydrogen diffusivity $\left(\log D-T^{-1}\right.$ plots). The activation energy, $E_{2}$ is determined from the slope by assuming the surface diffusion mechanism.

\section{CONCLUSIONS}

The diffusion behavior of gases in ultramicropore of MSCMs was studied in the present work. Based on the results of permeation experiment, mass transfer in MSCMs is considered to be complex flow of Knudsen diffusion and surface diffusion. Although the temperature dependence of permeation coefficient was apparently obey the Kunudsen diffusion in MSCMs which has wider ultramicropores, the deviation of separation factor, $\mathbf{P}_{\mathrm{H} 2} / \mathbf{P}_{\mathrm{D} 2}$, from the theoretical value was observed by using hydrogen and deuterium. In case of HTT1373K which has narrower ultramicropores, the size of which is comparable to hydrogen, the P-T ${ }^{-1 / 2}$ plots showed bad correlation, indicating surface 
diffusion dominantly occurs. The change of mass transfer mechanism is attributable to the shrinkage of pore width and the increase of physisorption potentials inside of pores. The linearity of the $\log \mathrm{D}-\mathrm{T}^{-1}$ plots was basically good and the activation energy was determined assuming the surface diffusion. The energy was high in narrow ultramicropores in HTT1373K and deuterium showed a higher value than hydrogen. The temperature dependence of the ratio of the diffusion coefficient, $\mathrm{D}_{\mathrm{H} 2} / \mathrm{D}_{\mathrm{D} 2}$, can be explained by physisorption; diffusivity of deuterium which has stronger physisorption potentials was lower than that of hydrogen. The apparent solubility in the bulk membrane was higher for denterium and the tendency remarkably appeared at low permeation temperatures where physisorption influences more effectively.

References

[1] Y. Yan, M. E. Davis and G. R. Gavalas, Ind. Eng. Chem. Res., 34, 1652 (1995).

[2] K. Kusakabe S. Yoneshige, A. Murata and S. Morooka, J. Membrane Sci., 116, 39-46 (1996).

[3] F. Kapteijin, W. J. W. Bakker, J. van de Graaf, G. Zheng, J. Poppe, J. A. Moulijin, Catalysis Today, 25, 213-218 (1995).

[4] N. K. Raman and C. J. Brinker, J. Membrane Sci., 273-279 (1995).
[5] B. N. Nair, K. Keizer, W. J. Elferink, M. J. Gilde, $H$. Verweij and A. J. Burggraaf, $J$. Membrane Sci., 116, 161-169 (1996).

[6] H. Hatori, Y. Yamada, M. Shiraishi, H. Nakata and S. Yoshitomi, Carbon, 30, 305-306 (1992).

[7] M. B. Rao and S. Sircar, J. Membrane Sci., 85, 253-264 (1993).

[8] H. Suda and K. Haraya, J. Chem. Soc., Chem. Commun., 1995, 1179-1180.

[9] S. Sircar, T. C. Golden and M. B. Rao, Carbon, 34, 1-12 (1996).

[10] V. C. Geiszler and W. J. Koros, Ind. Eng. Chem. Res., 35 , 2999-3003 (1996).

[11] J. Hayashi, H. Mizuta, M. Yamamoto, K. Kusakabe and S. Morooka, J. Membrane Sci., $124,243-251$ (1997).

[12] K. Kusakabe, M. Yamamoto, A. Morooka,J. Membrane Sci., 149, 59-67 (1998).

[13] H. Hatori, Y, Yamada, M. Shiraishi, H. Nakata S. Yoshitomi, M. Yoshihara and T. Kimura, TANSO, 1995 [No.167] 94-100.

[14] R. A. Lemons, J. Power Sources 29, 251-264 (1990).

[15] R. J. Bellows, E. P. Marucchi-Soons and D. T. Buckley, Ind. Eng. Chem. Res. 35, 1235-1242 (1996).

[16] H. Hatori, H. Takagi, Y. Yamada, Carbon 42, 1169-1173 (2004)

[17] R. M. Barrer, Trans. Faraday Soc. 35, 628-643 (1964).

(Received December 10, 2006;Accepted March 8, 2007) 